\title{
Some exact and numerical results for plane steady sheared flow of an incompressible inviscid fluid
}

\author{
Terry Brockett ${ }^{1}$ and Jung-Chun Suh ${ }^{2}$ \\ The Department of Naval Architecture and Marine Engineering, The Unicersity of Michigan, \\ Ann Arbor, MI 48109, USA \\ Received 8 February 1991
}

\begin{abstract}
Analytical and numerical solutions are presented for the steady flow of an inviscid fluid about symmetric lifting profiles at an angle of attack in a plane sheared onset flow for which conformal mapping plays a critical role. For uniform shear (i.e. the onset flow speed varies linearly with position) in two dimensions, the disturbance field is potential and hence a solution based on the conformal transformation technique may be constructed. The Moriya transformation, which employs a leading-term transformation coefficient that stretches and rotates the field at great distances from the foil (as distinct from other classical transformations which leave the far field unchanged) is used and, with a limited number of terms selected for the transformation, a simple elegant solution is obtained that may be easily evaluated at arbitrary points on the foil contour. An additional investigation is reported for the field solution - involving a locally similar but globally non-uniform sheared onset flow - about one of the foils for which a simple O-type grid is analytically generated from the mapping function. These data indicate that the uniform-shear solution overpredicts the lift and surface speed on the suction side of the foil relative to the more realistic onset flow: the numerical solution predicts surface speeds that generally lie between those for the uniform flow and the uniformly sheared flow solutions.
\end{abstract}

\section{Introduction}

Although many flow fields of practical interest are well represented by a potential disturbance to a uniform onset flow, a significant number of practical problems contain vorticity in the onset flow. For example, the classical turbomachinery problem of a rigid body rotating in a spatially non-uniform onset flow (e.g., rotor in a passage, multi-stage pumps and turbines, marine propeller in a hull wake) as well as airplanes and sailboats operating in the atmospheric boundary layer near the earth's surface cannot be accurately analyzed with potential theory alone. Treatment of these special flow problems requires complex numerical procedures to be applied and often accuracy is defined by comparisons with other numerical solutions or with simpler analytic flow fields. Even when the flow field is three-dimensional, a comparison with two-dimensional solutions is often presented for proof-of-concept validation. Here we present some simple results for the flow of an inviscid incompressible fluid about a symmetrical profile in a sheared onset flow. In general, the governing equations are the

Correspondence to: T. Brockett, Areté Associates Inc., P.O. Box 16287, Arlington, VA 22215, USA.

1 Present address: Areté Assoc., P.O. Box 16287, Arlington VA 22215, USA.

2 Present address: Korea Research Institute of Ships and Ocean Engineering, P.O. Box 1, Deaduk Danji, Daejeon 305-606, Korea. 


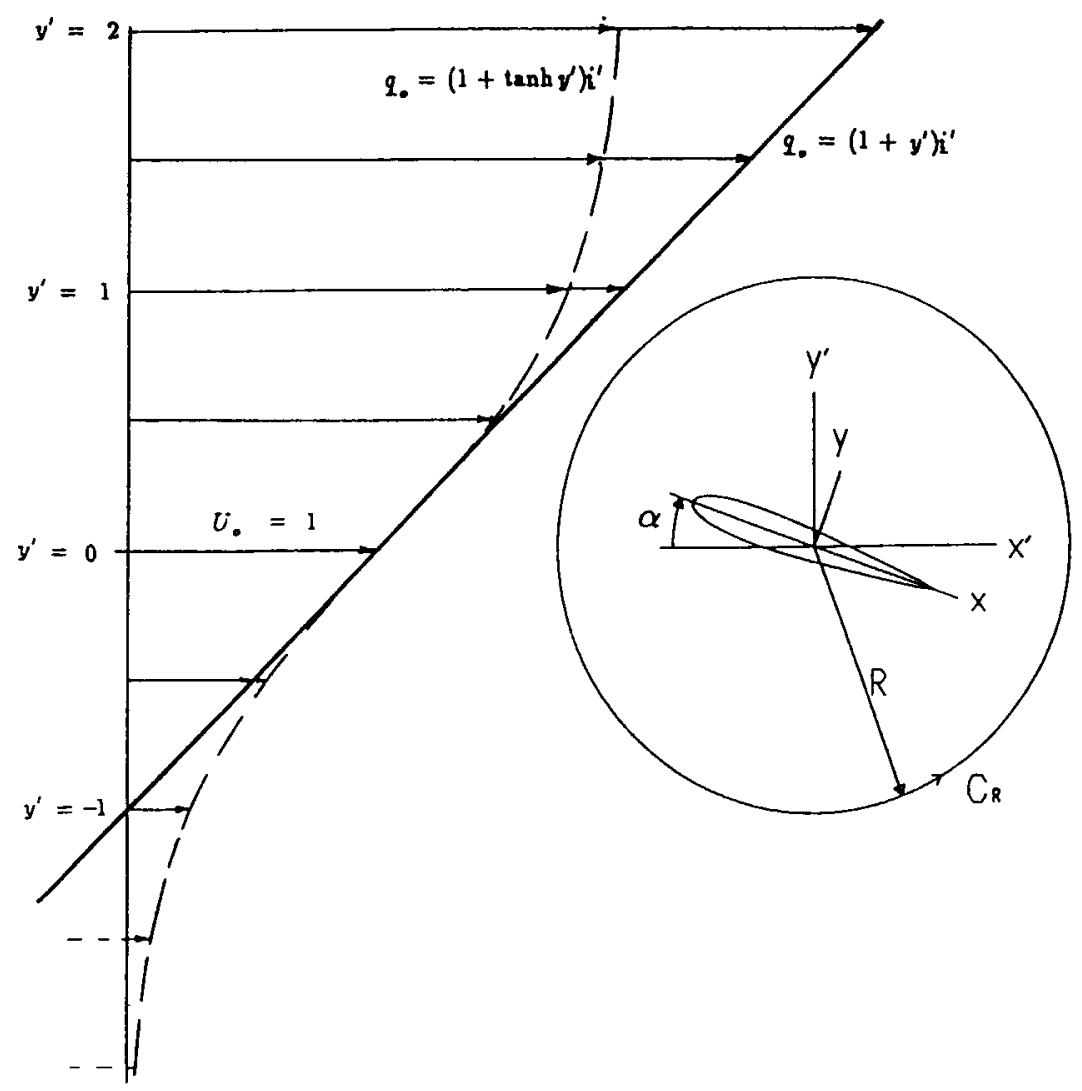

Fig. 1. Flow field schematic for sheared onset flow.

continuity and the Navier-Stokes (or vorticity transport) equations. When the fluid is incompressible and inviscid and the fields is two-dimensional, the vorticity equation is

$$
\mathrm{D} \omega / \mathrm{D} t=0
$$

where the substantial derivative is indicated with the capital letters and $\omega$ is the scalar plane component of the vorticity vector, $\omega=\nabla \times q=\omega k$. This vorticity equation states that the vorticity of a particle is unchanged as the particle is convected with the field velocity. For the special case of a linearly varying onset flow, say one at an angle $\alpha$ relative to the nose-tail line with the free-stream velocity defined relative to a coordinate system aligned with the nose-tail line as (see sketch in fig. 1):

$$
\boldsymbol{q}_{0}=\left[U_{0}+K(y \cos \alpha-x \sin \alpha)\right] \boldsymbol{e}_{\alpha},
$$

where $e_{\alpha}=i^{\prime}=\cos \alpha i+\sin \alpha j$, the vorticity is constant

$$
\boldsymbol{\omega}=\omega_{0} \boldsymbol{k}=-K \boldsymbol{k}
$$

and hence any disturbance must be a potential one since there can be no change in the value of vorticity as a particle moves into a region where there is a velocity component in addition to the onset flow. Thus we may take the velocity vector to be

$$
\boldsymbol{q}=\boldsymbol{q}_{0}+\nabla \phi_{1}=\boldsymbol{q}_{0}+\boldsymbol{u}_{p}
$$

where $\phi_{1}$ is the disturbance potential (and $u_{p}=\nabla \phi_{1}$ is the perturbation velocity) that tends to 
a constant value far from the profile. From the continuity equation, the disturbance potential is governed by the Laplace equation

$$
\nabla \cdot u_{p}=\Delta^{2} \phi_{1}=0
$$

Solution of this equation, subject to the boundary conditions, is our first problem. Once the solution is found, the loads may be derived from a modified Bernoulli equation. As shown by Yih (1969), an appropriate integration of the governing dynamical equation gives:

$$
p / p+\frac{1}{2} q^{2}+\omega_{0} \Psi=\text { constant everywhere, }
$$

where $\Psi=\Psi_{0}+\psi_{1}$ is the streamfunction of the flow; i.e. $\boldsymbol{q}=\boldsymbol{\nabla} \times(\Psi \boldsymbol{k})$. On the profile contour, the value of $\Psi$ is fixed and hence the third term of the modified Bernoulli equation merely adds a constant to the pressure. For a lifting foil, the body (or dividing) streamline may be a closed loop within the field or may be displaced to a great distance from the body (as it may be in pure potential flow also because of a logarithmic component in the perturbation streamfunction $\psi_{1}$ associated with the circulation). For our simple onset flow, the streamfunction far from the body tends to

$$
\Psi_{0}=U_{0}(y \cos \alpha-x \sin \alpha)+K\left(\frac{1}{2} y^{2} \cos ^{2} \alpha+\frac{1}{2} x^{2} \sin ^{2} \alpha-x y \sin \alpha \cos \alpha\right)+H,
$$

where $H$ is a constant. Thus when the body streamline extends to great distances from the body (for which the streamline position $y$ may tend to $\pm \infty$ ), there may be an ambiguity in the reference level of the pressure. This ambiguity has been discussed by Tsien (1943), Atassi (1984) and others and we do not repeat their comments. If we take the pressure and reference speed to be $p_{\infty}$ and $U_{0}$ and the streamfunction to be 0 at some far-upstream point along $y=x \tan \alpha$, then the pressure coefficient is:

$$
C_{p}=\frac{p-p_{\infty}}{\frac{1}{2} \rho U_{0}^{2}}=1-\frac{2 \omega_{0} \Psi_{\mathrm{B}}}{U_{0}^{2}}-\frac{q \cdot \boldsymbol{q}}{U_{0}^{2}}
$$

which, to within an additive constant - that is a multiple of the (perhaps infinite) value of the streamfunction on the body, $\Psi_{\mathrm{B}}-$ is the familiar Bernoulli equation:

$$
C_{p}=1-\boldsymbol{q} \cdot \boldsymbol{q} / U_{0}^{2} \text {. }
$$

A knowledge of this traditional pressure coefficient is sufficient to determine loads on the profile.

The problem just defined is one addressed by Tsien (1943) using the Joukowsky (1910) transformation. Tsien found a significant shift in the angle of zero lift that varies almost linearly with the gradient of the onset flow. One limit of our family of profiles is similar to his shape and our results for it are quite similar in trend. However our solution encompasses a family of profiles and is in a form readily accessible at arbitrary points.

The basic procedure in a conformal transformation is to map the flow about a simple body into that about a more complex shape. One shape for which a solution can be readily defined is the circular cylinder. When the solution field is potential, a conformal mapping or transformation will provide the solution about another body. This process is addressed in many books on aerodynamics (e.g., Ashley and Landahl, 1965). One such transformation is that proposed by Moriya (1938, 1941), which has recently been revisited by Brockett (1989a). In the Moriya mapping, the region at great distances from the foil is stretched and rotated by taking the leading term in the mapping to have a complex-constant multiple. This additional feature is useful when the mapping is restricted to only a few terms, in which case an additional condition can be met with this leading coefficient. In particular, the chord of the transformed foil can be set to a fixed value (Brockett, 1989a), rather than being dependent on 
the mapping parameters. A simple relation is also found for stations on the two fields (cylinder and foil). This simplicity in point specification is desirable when making quantitative comparisons between numerical and analytical solutions.

Although these data provide information for comparison with numerical methods and on the general influence of onset shear on surface speeds and loads, the analytic results may be somewhat different than the solution for locally similar non-uniform onset profiles. Murray and Mitchell (1957) have shown that the uniform shear results are not an adequate representation for the non-uniform shear flow about a non-lifting circular cylinder. Also Payne and Nelson (1985) have experimentally evaluated an $18 \%$ thick profile in a wind tunnel with a non-dimensional shear gradient that is about $K=0.6$ but with limited vertical extent of the field. They found the shape of the pressure coefficient curves was not altered greatly (and argued that the velocity gradient had a negligible effect on the aerodynamic load coefficients) but the magnitude of the absolute pressure was changed. The Reynolds number of their tests was from 0.75 to $2 \times 10^{5}$ and hence together with severe adverse gradients associated with the aft end of the thick foil may have not produced results that are similar to fully attached turbulent flow. However, in combination with the analysis of Murray and Mitchell (1957) these experiments do suggest that an unbounded uniform shear flow may not be an adequate representation of real-flow situations. Hence a numerical solution has been undertaken to define the flow about one of the symmetrical profiles previously defined with the Moriya transformation. For this investigation, the conformal transformation of the flow field provides a simple procedure for defining a grid mesh for a finite-element field solution. A simple grid is specified in the circle plane and then mapped to one appropriate for the field about the foil. The simple grid may be a $\mathrm{H}$-type grid if we select a set of irrotational streamlines and potential lines in the circle plane or may be an O-type grid if we select concentric circles and rays as the grid in the circle plane. Here we select the O-type grid but believe the H-type will work as well. Our solution procedure is one that subdivides the perturbation velocity component that arises in the flow with non-uniform shear into two components: one potential and one rotational. Specifically we define the three components of the total velocity vector:

$$
\boldsymbol{q}=\boldsymbol{q}_{0}+\boldsymbol{u}_{\mathrm{p}}+\boldsymbol{u}_{\mathrm{s}}
$$

where $\boldsymbol{q}_{0}$ is the specified non-uniform onset velocity vector, $\boldsymbol{u}_{\mathrm{p}}$ is the (potential) perturbation response when the velocity vector $\left(\boldsymbol{q}_{0}+\boldsymbol{u}_{\mathrm{s}}\right)$ is known ${ }^{\# 1}$, and $\boldsymbol{u}_{\mathrm{s}}$ is the velocity term associated with the vorticity dynamics of the problem. In this case, eq. (1) is used to define the vorticity field vector $\omega_{\mathrm{s}}$ associated with the perturbation velocity component $\boldsymbol{u}_{\mathrm{s}}$ (i.e, $\omega=\omega_{0}+\omega_{\mathrm{s}}$ ). This approach has been proposed by Brockett (1986) for three-dimensional problems and is herein evaluated for two-dimensional flows. We use the fundamental vector identity to define the perturbation velocity $u(x)=u_{\mathrm{p}}(x)+u_{\mathrm{s}}(x)$

$$
\begin{aligned}
u(x)= & \frac{1}{2 \pi} \oint_{\mathrm{C}}\left([n(s) \times u(s)]+\frac{x-s}{|x-s|^{2}}+n(s) \cdot u(s) \frac{x-s}{|x-x|^{2}}\right) \mathrm{d} l_{\mathrm{s}} \\
& +\frac{1}{2 \pi} \iint_{\mathrm{S}} \frac{\omega_{\mathrm{s}} k \times(x-s)}{|x-s|^{2}} \mathrm{~d} S,
\end{aligned}
$$

where $n$ points into the field and the third (area) integral specifically defines the velocity component $\boldsymbol{u}_{\mathrm{s}}$ as an improper integral with integrable singularity at $\boldsymbol{x}$. The vorticity vector $\boldsymbol{\omega}_{\mathrm{s}}$ is obtained from eq. (1) by iteration for a known $\boldsymbol{u}_{\mathrm{p}}$. Once the total velocity vector is known, the pressure can be defined on the body contour to within an additive constant by eq. (9). We

\# 1 The combination $\boldsymbol{q}_{0}+\boldsymbol{u}_{\mathrm{s}}$ is sometimes defined as the effective onset flow since if it can be defined or estimated, only potential analysis is required to find a solution. 
select a finite-volume time-marching scheme to solve eq. (1) using an initial condition $\boldsymbol{u}_{\mathrm{s}}=\mathbf{0}$, with occasional updates of the total perturbation velocity to include a computation of $\boldsymbol{u}_{\mathrm{s}}$ from the area integral. We note that once a stable solution approach has been found based on this vorticity-velocity equation it can be directly extended to a non-inertial reference frame (Speziale, 1987). Herein, we outline our solution procedure and refer to Suh (1990) for further details.

We present as results the analytical solution for the flow field about a class of symmetrical profiles derived from the Moriya transformation applied to the solution for a uniformly sheared onset flow about a circular cylinder. The surface speeds as well as the loads are derived. Selected streamline patterns are presented. In addition, numerical data for a field solution about one of these foils in non-uniform shear (using the O-type grid described previously) are presented to illustrate the difference between the uniformly sheared onset and a non-linear sheared one that is locally similar. In fig. 1 the general orientation and coordinate systems for the flow about the foil are shown. Two onset profiles are illustrated, one with a uniform gradient $\left(q_{0}=U_{0}\left(1+y^{\prime}\right)\right)$ and the other $\left(q_{0}=U_{0}\left(1-\tanh y^{\prime}\right)\right)$ with a similar local gradient but tending to constant values far to the sides of the foil. This second onset profile does not meet the conditions for a potential disturbance and the numerical field solution procedure just outlined must be applied. A feature of our solution for non-uniform shear is a reduction in the maximum value of the surface speed and a reduced lift compared to a locally similar uniformly sheared onset flow.

\section{Solution for uniform shear by conformal transformation}

The general Moriya transformation is specified as

$$
z=x+\mathrm{i} y=C_{-1} \zeta / a+C_{0}+\sum_{n=1}^{\infty} C_{n} a^{n} / \zeta^{n},
$$

where $z=x+\mathrm{i} y$ is the complex coordinate of a point in the physical plane ( $z$-plane), $\zeta=\xi+\mathrm{i} \eta$ is the complex coordinate in the circle plane ( $\zeta$-plane), $a$ is a characteristic length dimension (taken as the radius of a circle that is set to 1 for non-dimensionalization), and $C_{n}=A_{n}+\mathrm{i} B_{n}$ are complex mapping constants. We consider only the case that a circle of radius $a$ is transformed into a family of symmetric profiles (for which all $B_{n}$ are zero). The coefficients $A_{n}(n \leq 2)$ may be expressed in terms of a two-parameter family $(\epsilon, \delta)$ be invoking both closure and that the profile chordlength extent between the leading edge, LE, and the trailing edge, TE, be unity (Brockett, 1989a):

$$
A_{-1}=(1+2 \epsilon) / 4, \quad A_{0}=-\epsilon \delta, \quad A_{1}=(1-2 \epsilon) / 4, \quad A_{2}=\epsilon \delta .
$$

Then the coordinates $(x, y)$ of an arbitrary point in the physical plane can be written in terms of the polar coordinates $(r, \theta)$ in the circle plane as:

$$
\begin{aligned}
& x(r, \theta)=A_{0}+\left(A_{-1} r+A_{1} / r\right) \cos \theta+A_{2} / r^{2} \cos 2 \theta, \\
& y(r, \theta)=\left(A_{-1} r-A_{1} / r\right) \sin \theta-A_{2} / r^{2} \sin 2 \theta,
\end{aligned}
$$

for which the profile shape $(r=a)$ is

$$
\begin{aligned}
& x(a, \theta) \equiv x(\theta)=\frac{1}{2} \cos \theta+\epsilon \delta(\cos 2 \theta-1), \\
& y(a, \theta) \equiv y(\theta)=\epsilon(\sin \theta-\delta \sin 2 \theta) .
\end{aligned}
$$

The coordinates of the TE and LE correspond, respectively, to $(0.5,0)$ and $(-0.5,0)$ in the physical plane, and $(1,0)$ and $(-1,0)$ in the circle plane. For $\delta=0$, the profile shape is an 
ellipse with thickness ratio $\tau=2 \epsilon$. A profile with a cusped trailing edge similar to a Joukowsky foil can be obtained by taking $\delta=0.5$, otherwise (i.e. $0<\delta<0.5$ ) the trailing edge has a round shape. The thickness ratio is given by (if $\delta \neq 0$ )

$$
\tau=\left[\epsilon\left(3+\sqrt{1+32 \delta^{2}}\right) / 16 \delta\right]\left(32 \delta^{2}-2+2 \sqrt{1-32 \delta^{2}}\right)^{1 / 2}
$$

at the location $x=\left[(1+\epsilon)-\left(1-\left(1+32 \delta^{2}\right)^{1 / 2}\right] / 16 \delta-\epsilon \delta\right.$. The parameter $\epsilon$ is associated predominantly with the thickness of profiles and $\delta$ predominately with the trailing-edge shape. The coordinates and surface speed are readily determined at arbitrary chordwise stations since the relation between $x$ and $\theta$ can be inverted (Brockett, 1989a):

$$
\theta(x)=\cos ^{-1}\left(\frac{\sqrt{1+16 \delta \epsilon(2 x+4 \epsilon \delta)}-1}{8 \delta \epsilon}\right) .
$$

The complex (disturbance) potential $F_{1}(\zeta)$ can be assumed in a form that satisfies the Laplace equation and the required far-field behavior:

$$
F_{1}(\zeta)=\phi_{1}+\mathrm{i} \psi_{1}=-\mathrm{i}\left(\Gamma_{\mathrm{B}} / 2 \pi\right) \ln \zeta+c_{1} \zeta^{-1}+c_{2} \zeta^{-2}+\cdots,
$$

where $\phi_{1}$ is the disturbance potential and $\psi_{1}$ is the disturbance stream function. Here the (disturbance) bound circulation is $\Gamma_{\mathrm{B}}$ (with positive direction counterclockwise) and the complex coefficients $c_{n}=\alpha_{n}+\mathrm{i} \beta_{n}$ of the potential function $F_{1}(\zeta)$ are to be selected such that the kinematic body boundary condition and the Kutta condition at the TE are met.

The kinematic boundary condition $\left(\left.\boldsymbol{q} \cdot \boldsymbol{n}\right|_{\text {body }}=0\right)$ transformed to the circle in the $\zeta$-plane becomes $\# 2$

$$
\left.r^{-1} 1 \partial \psi_{1} /\left.\partial \theta\right|_{r=1}=-\boldsymbol{q}_{0} \cdot \boldsymbol{n}|\mathrm{d} z / \mathrm{d} \zeta|\right)\left.\right|_{\zeta=\mathrm{e}^{\mathrm{i} \theta}}
$$

where the undisturbed (sheared onset) velocity $\boldsymbol{q}_{0}$ in the $z$-plane is given by eq. (2). From eq. (19) the unknown coefficients $c_{n}$ in the potential, $\alpha_{n}$ and $\beta_{n}$ (made non-dimensional by the chordlength $c$, the radius of the circle $a$ and the reference speed $U_{0}$ ), are determined; the coefficients with $n$ greater than 4 in eq. (18) being zero:

$$
\begin{aligned}
\alpha_{1}= & \left(A_{-1}-A_{1}\right) \cos \alpha+\frac{1}{2} K\left(A_{-1} A_{2}-A_{0} A_{-1}+A_{0} A_{1}\right) \sin 2 \alpha, \\
\beta_{1}= & \left(A_{-1}+A_{1}\right) \sin \alpha \\
& +\frac{1}{2} K\left[A_{2}\left(A_{-1}-A_{1}\right) \cos ^{2} \alpha-\left(A_{-1}+A_{1}\right)\left(2 A_{0}+A_{2}\right) \sin ^{2} \alpha\right], \\
\alpha_{2}= & -A_{2} \cos \alpha-\frac{1}{4} K\left(A_{-1}^{2}-A_{1}^{2}-2 A_{0} A_{2}\right) \sin 2 \alpha, \\
\beta_{2}= & A_{2} \sin \alpha+\frac{1}{4} K\left\{\left(A_{-1}-A_{1}\right)^{2} \cos ^{2} \alpha-\left[\left(A_{-1}+A_{1}\right)^{2}+4 A_{0} A_{2}\right] \sin ^{2} \alpha\right\}, \\
\alpha_{3}= & \frac{1}{2} K A_{1} A_{2} \sin 2 \alpha, \quad \beta_{3}=\frac{1}{2} K\left(A_{1} A_{2} \cos 2 \alpha-A_{-1} A_{2}\right), \\
\alpha_{4}= & \frac{1}{4} K A_{2}^{2} \sin 2 \alpha, \quad \beta_{4}=\frac{1}{4} K A_{2}^{2} \cos 2 \alpha .
\end{aligned}
$$

Now, to find $\Gamma_{\mathrm{B}}$, a stagnation point should be imposed at the TE (i.e. the Kutta condition is invoked):

$$
\left.\left(-\partial \psi_{1} / \partial r+\boldsymbol{q}_{0} \cdot \boldsymbol{t}|\mathrm{d} z / \mathrm{d} \zeta|\right)\right|_{r=1, \theta=0}=0 .
$$

Inserting (20) for the coefficients $\alpha_{n}$ and $\beta_{n}$ into (21) and solving for $\Gamma_{\mathrm{B}}$ (non-dimensionalized by $U_{0}$ and $c$ ), we find

$$
\Gamma_{\mathrm{B}} / 2 \pi=-\left(\sum_{n=1}^{4} n \beta_{n}+\left(A_{-1}-A_{1}-2 A_{2}\right)\left(1-\frac{1}{2} K \sin \alpha\right) \sin \alpha\right) \text {. }
$$

\footnotetext{
\#2 Note that because of the rhs of eq. (19), the solution in the $\zeta$-plane contains information about the profile rather than being a universal field as in full potential flow.
} 
The total surface speed $q_{\mathrm{s}}=\left.\boldsymbol{q} \cdot \boldsymbol{t}\right|_{\text {body }}$ in the direction of increasing $\theta$ is

$$
q_{\mathrm{s}}(\theta)=\left.\boldsymbol{q}_{0} \cdot \boldsymbol{t}\right|_{\text {body }}-\frac{\partial \psi_{1} / \partial r}{|\mathrm{~d} z / \mathrm{d} \zeta|_{r=1}},
$$

which can be expressed in terms of profile parameters $(\epsilon, \delta)$ as:

$$
\begin{aligned}
q_{\mathrm{s}} / U_{0}= & \left(d_{0}+d_{1} \sin \theta+d_{2} \cos \theta+d_{3} \sin 2 \theta+d_{4} \cos 2 \theta+d_{5} \cos 3 \theta\right) \\
& \times\left[\left(\frac{1}{2} \sin \theta+2 \epsilon \delta \sin 2 \theta\right)^{2}+\epsilon^{2}(\cos \theta-2 \delta \cos 2 \theta)^{2}\right]^{-1 / 2}
\end{aligned}
$$

where

$$
\begin{aligned}
& d_{0}=-\left(\frac{1}{2}+\epsilon\right) \sin \alpha+K\left[\epsilon^{2}\left(\delta-\frac{1}{2}\right)-\frac{1}{4} \epsilon+\left(\frac{1}{8}+\frac{1}{2} \epsilon-\frac{1}{2} \epsilon \delta+\frac{1}{2} \epsilon^{2}-\epsilon^{2} \delta\right) \sin ^{2} \alpha\right], \\
& d_{1}=-\left(\frac{1}{2}+\epsilon\right) \cos \alpha-K \epsilon \delta\left(\epsilon+\frac{1}{2}\right) \cos \alpha \sin \alpha, \\
& d_{2}=\left(\frac{1}{2}+\epsilon\right) \sin \alpha+K \epsilon \delta\left[\left(\frac{1}{4}-\frac{1}{2} \epsilon\right) \cos ^{2} \alpha+\left(\frac{1}{2} \epsilon+\frac{3}{4}\right) \sin ^{2} \alpha\right], \\
& d_{3}=K\left(\frac{1}{8}+\frac{1}{2} \epsilon+\frac{1}{2} \epsilon^{2}\right) \cos \alpha \sin \alpha, \\
& d_{4}=K\left[\left(\frac{1}{4} \epsilon+\frac{1}{2} \epsilon^{2}\right) \cos ^{2} \alpha-\left(\frac{1}{8}+\frac{1}{4} \epsilon\right) \sin ^{2} \alpha\right], \\
& d_{5}=-K \epsilon \delta\left(\frac{1}{2} \epsilon+\frac{1}{4}\right) .
\end{aligned}
$$

To easily obtain a closed form expression for loads, we use expressions obtained from the momentum relations for the field, which produce integrals over only the contour bounding the field at a large radius $(R)$, as in Tsien (1943) (see fig. 1). The force components $F_{x}$ and $F_{y}$ (in the $x$-and $y$-direction of the coordinate system fixed on the foil) and the moment $M_{0}$ acting on the foil (about the origin or foil midchord) are expressed as integrals of the pressure and velocity distribution on the circle $\mathrm{C}_{R}$ enclosing the field. The following integrals express the conservation theorem for momentum and angular momentum:

$$
\begin{aligned}
& F_{x}=-\int_{\mathrm{C}_{R}} p \mathrm{~d} y-\int_{\mathrm{C}_{R}} \rho q_{x}\left(q_{x} \mathrm{~d} y-q_{y} \mathrm{~d} x\right), \\
& F_{y}=\int_{\mathrm{C}_{R}} p \mathrm{~d} x-\int_{\mathrm{C}_{R}} \rho q_{y}\left(q_{x} \mathrm{~d} y-q_{y} \mathrm{~d} x\right), \\
& M_{0}=\int_{\mathrm{C}_{R}} p(x \mathrm{~d} x+y \mathrm{~d} y)-\int_{\mathrm{C}_{R}} \rho\left(x q_{y}-y q_{x}\right)\left(q_{x} \mathrm{~d} y-q_{y} \mathrm{~d} x\right),
\end{aligned}
$$

where $q_{x}$ and $q_{y}$ are, respectively, the $x$ - and $y$-component of the total velocity on the contour $\mathrm{C}_{R}$ in the physical plane. To apply this equation, the complex (disturbance) velocity potential should be expressed in terms of $z$ instead of $\zeta$ using an inverse of the mapping function. The inversion of the Moriya transformation (eqs. 12,14) given herein is readily found as a power series of $z$. We need terms up to only $1 / z^{2}$ because the contribution from terms greater than this second (negative) power to the integrals in (25) will vanish as the radius of the enclosing circle $(R)$ tends to infinity. The inverse mapping in turn allows us to express the complex (disturbance) velocity potential (eq. 18) as a power series $z$ :

$$
\begin{aligned}
F_{1}(z)= & -\mathrm{i}\left(\Gamma_{\mathrm{B}} / 2 \pi\right) \log \left(z / A_{-1}\right)+\left[\mathrm{i}\left(\Gamma_{\mathrm{B}} / 2 \pi\right) A_{0}+\left(\alpha_{1}+\mathrm{i} \beta_{1}\right) A_{-1}\right] z^{-1} \\
& +\left[\mathrm{i}\left(\Gamma_{\mathrm{B}} / 2 \pi\right)\left(A_{-1} A_{1}+\frac{1}{2} A_{0}^{2}\right)+A_{-1} A_{0}\left(\alpha_{1}+\mathrm{i} \beta_{1}\right)\right. \\
& \left.+A_{-1}^{2}\left(\alpha_{2}+\mathrm{i} \beta_{2}\right)\right] z^{-2}+\cdots
\end{aligned}
$$


Now using (26), together with the coefficients from (20), for the velocity components and (6) for the pressure, we find the lift and moment coefficients (about the midchord point):

$$
\begin{aligned}
& C_{\mathrm{L}}=\left(F_{y} \cos \alpha+F_{x} \sin \alpha\right) / \frac{1}{2} \rho U_{0}^{2} c=4 \pi\left(a_{0}-K b_{1}\right), \\
& C_{\mathrm{M}}=M_{0} / \frac{1}{2} \rho U_{0}^{2} c^{2}=-4 \pi\left(a_{1}+K b_{2}\right)
\end{aligned}
$$

where (recall that $\Gamma_{\mathrm{B}}, A_{n}, \alpha_{n}$, and $\beta_{n}$ have been non-dimensionalized by $U_{0}, c$ and $a$ ):

$$
\begin{aligned}
a_{0}= & -\Gamma_{\mathrm{B}} / 2 \pi, \\
a_{1}= & {\left[\left(\Gamma_{\mathrm{B}} / 2 \pi\right) A_{0}+A_{-1} \beta_{1}\right] \cos \alpha-A_{-1} \alpha_{1} \sin \alpha, } \\
b_{1}= & -\left[\left(\Gamma_{\mathrm{B}} / 2 \pi\right) A_{0}+A_{-1} \beta_{1}\right] \sin \alpha-A_{-1} \alpha_{1} \cos \alpha, \\
b_{2}= & -\left(A_{-1} A_{0} \alpha_{1}+A_{-1}^{2} \alpha_{2}\right) \cos 2 \alpha \\
& -\left[\left(\Gamma_{\mathrm{B}} / 2 \pi\right)\left(A_{-1} A_{1}+\frac{1}{2} A_{0}^{2}\right)+A_{-1} A_{0} \beta_{1}+A_{-1}^{2} \beta_{2}\right] \sin 2 \alpha .
\end{aligned}
$$

For an ellipse of thickness ratio $\tau=2 \epsilon$ (i.e., $\delta=0$ ), the quantities $q_{\mathrm{s}}, C_{\mathrm{L}}$ and $C_{\mathrm{M} 0}$ can be expressed simply in terms of $K, \alpha, \tau$ as:

$$
\begin{aligned}
& \frac{q_{\mathrm{s}}}{U_{0}}= \frac{e_{0}+e_{1} \sin \theta+e_{2} \cos \theta+e_{3} \sin 2 \theta+e_{4} \cos 2 \theta}{\sqrt{\sin ^{2} \theta+\tau^{2} \cos ^{2} \theta}}, \\
& C_{\mathrm{L}}=\pi\left[2(1+\tau) \sin \alpha+K \tau\left(\tau \cos ^{2} \alpha-\sin ^{2} \alpha+\frac{1}{2}\right)\right], \\
& C_{\mathrm{M} 0}=\frac{1}{64} \pi\left(1-\tau^{2}\right) \sin 2 \alpha[16+8 K(1+\tau) \sin \alpha \\
&\left.\quad+K^{2}(1+3 \tau)\left(\tau \cos ^{2} \alpha-\sin ^{2} \alpha\right)\right],
\end{aligned}
$$

where

$$
\begin{aligned}
& e_{0}=-(1+\tau)\left[\sin \alpha+\frac{1}{4} K\left(\tau \cos ^{2} \alpha-\sin ^{2} \alpha\right)\right], \quad e_{1}=-(1+\tau) \cos \alpha, \\
& e_{2}=(1+\tau) \sin \alpha, \quad e_{3}=\frac{1}{4} K(1+\tau)^{2} \cos \alpha \sin \alpha, \\
& e_{4}=\frac{1}{4} K(1+\tau)\left(\tau \cos ^{2} \alpha-\sin ^{2} \alpha\right) .
\end{aligned}
$$

From eq. (30), the increment in lift coefficient due to the sheared onset flow becomes $\pi K \tau\left(\frac{1}{2}+\tau-\left(1+\frac{1}{2} \tau\right) \alpha^{2}+\cdots\right)$. It follows that the lift force increment increases with both increasing shear gradient and thickness of the ellipse but decreases slightly as the angle of attack departs from zero. From (30), the angle of attack at zero-lift for an ellipse in uniform shear flow is given by

$$
\alpha_{0}=\sin ^{-1}\left[(1 / K \tau)\left(1-\sqrt{1+(K \tau)^{2}(2 \tau+1) / 2(\tau+1)}\right)\right]
$$

which becomes approximately $-K \tau / 4$ (in radians) for small values of $\tau$. Thus there is a shift of the zero-lift angle of attack due to shear flow that is nearly linearly proportional to both $K$ and $\tau$. The shear flow terms in the moment coefficient (eq. 31) are small for normal $\alpha$ and $\tau$ values.

Another feature due to shear flow is changes in the streamline patterns and velocity components. The shape of streamlines can be obtained by fixing the value of the stream function. Typically, the non-dimensional value of the stream function $\left(\Psi=\Psi_{0}+\psi_{1}\right.$, the sum of the stream function of the onset and disturbed flow) is assigned a constant value $C_{\psi}$,

$$
\begin{aligned}
C_{\psi}= & \left(y_{\mathrm{v}} \cos \alpha-x_{\mathrm{v}} \sin \alpha\right)+\frac{1}{2} K\left(y_{\mathrm{v}} \cos \alpha-x_{\mathrm{v}} \sin \alpha\right)^{2}-\left(\Gamma_{\mathrm{B}} / 2 \pi\right) \ln r_{\mathrm{v}} \\
& +\sum_{n=1}^{4}\left(-\alpha_{n} \sin n \theta_{\mathrm{v}}+\beta_{n} \cos n \theta_{\mathrm{v}}\right) / r_{\mathrm{v}}^{n},
\end{aligned}
$$


(o)

$$
c=0.05, \delta=0
$$

(b)

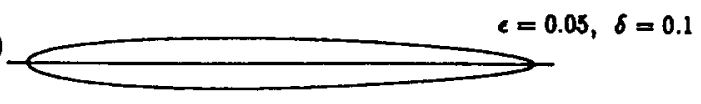

(c)

$\epsilon=0.05, \delta=0.3$

(d)

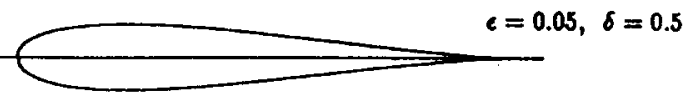

(e)

$\epsilon=0.01, \delta=0.4$

(t)

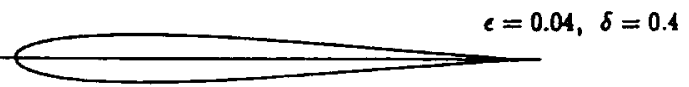

(g)

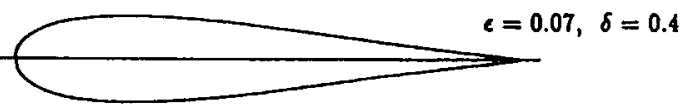

(h)

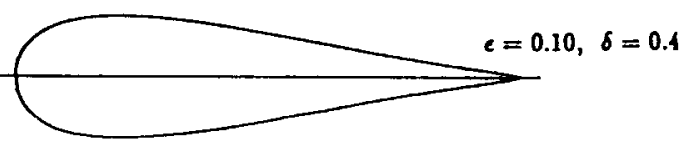

Fig. 2. Typical profiles specified by the Moriya transformation.

(a)

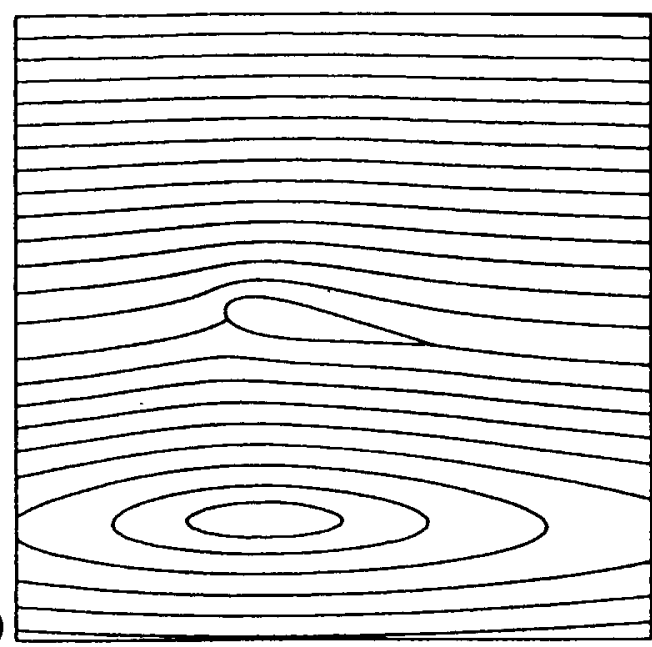

(b)

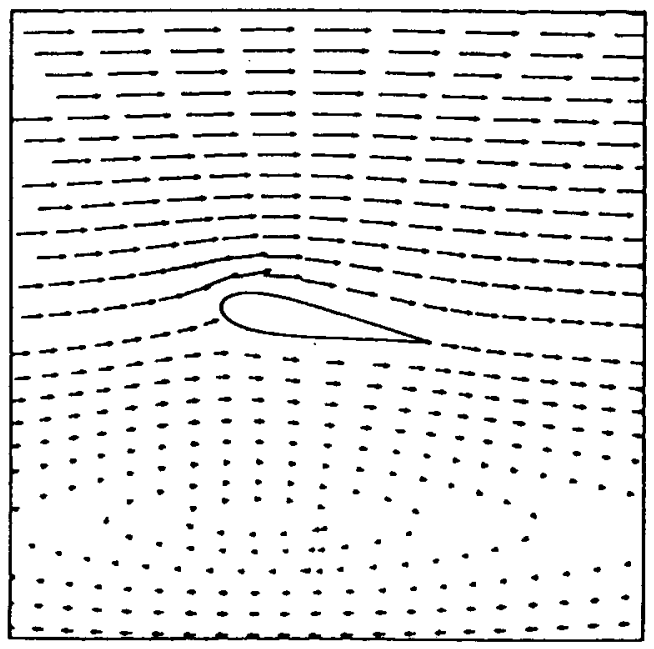

Fig. 3. Streamline shape and velocity distribution about a Moriya foil $(\epsilon=0.075, \delta=0.4)$ in steady shear flow $(K=1)$. The angle of attack $\alpha$ is $10^{\circ}$. (a) Streamlines, (b) velocity. 


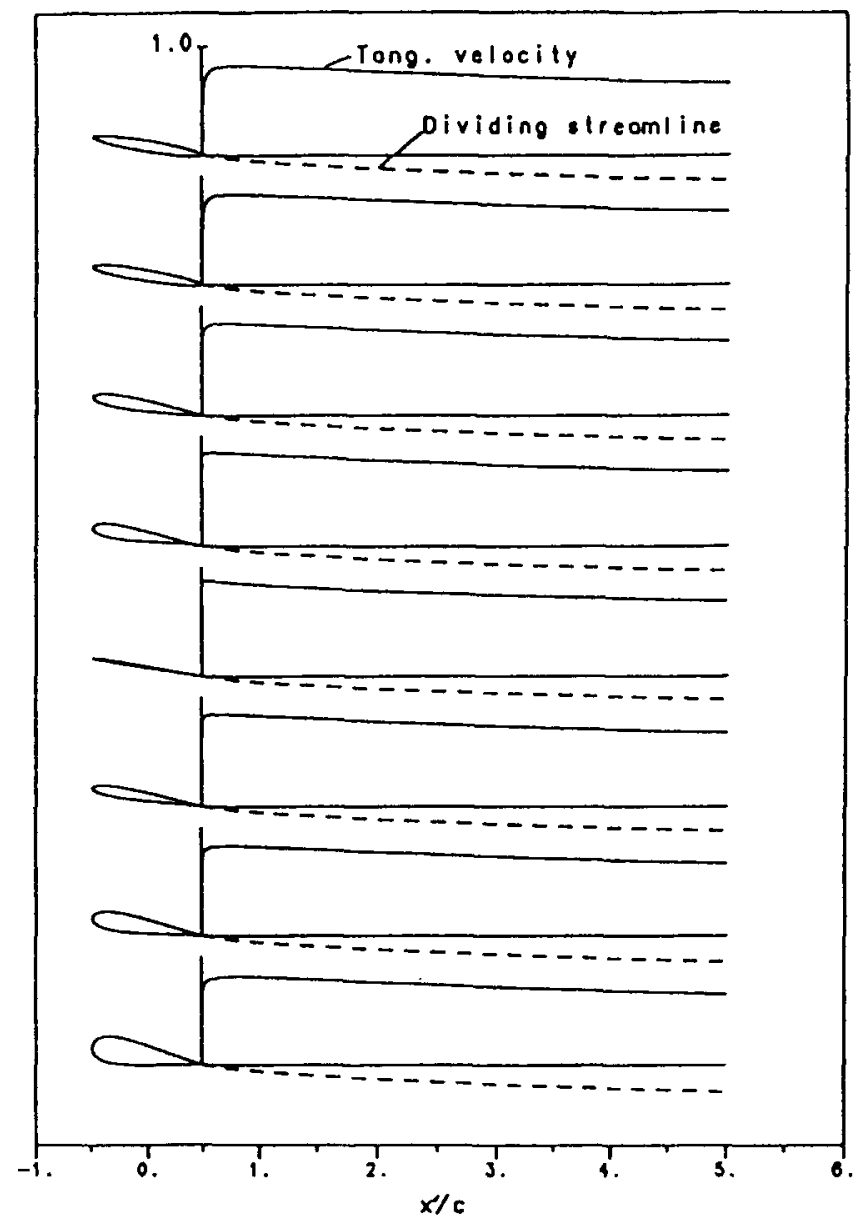

Fig. 4. Dividing trailing edge streamline and tangential velocity component along it for Moriya foils in uniform shear flow $(K=1)$. The foils are the same in order (from top to bottom) as ones in fig. 2. The angle of attack $\alpha$ is $10^{\circ}$ for all cases.

where $\left(r_{v}, \theta_{v}\right)$ are the polar coordinates of a point $\left(\zeta_{v}=\xi_{v}+i \eta_{v}\right)$ on the streamline in the circle plane and $\left(x_{\mathrm{v}}, y_{\mathrm{v}}\right)$ are Cartesian coordinates of the corresponding point in the physical plane. The disturbance velocity vector $\boldsymbol{u}_{\mathrm{p}}$ in the field is

$$
u_{x}-\mathrm{i} u_{y}=\left.\frac{\mathrm{d} F_{1} / \mathrm{d} \zeta}{\mathrm{d} z / \mathrm{d} \zeta}\right|_{\zeta=r \mathrm{e}^{i \theta}},
$$

which can be obtained in terms of the polar coordinates $(r, \theta)$ of a point on a streamline by using (18) and (33). Adding the undisturbed velocity components we obtain the total resultant velocity. Figure 3 illustrates these calculated results for streamlines and velocity components. As expected, significant changes in the streamline shape and velocity components relative to those of uniform flow (although not shown here) are found near the region where the velocity reverses direction (see the "swirl" in fig. 3). For each of the foil sections show in fig. 2, the dividing (trailing-edge) streamline and tangential velocity component along it are presented in fig. 4. All tangential velocity distributions are smooth along the downstream dividing streamlines except for a rapid change near the TE where the flow recovers from a stagnation point. Also the dividing streamline in the field and the tangential velocity distribution along it are 


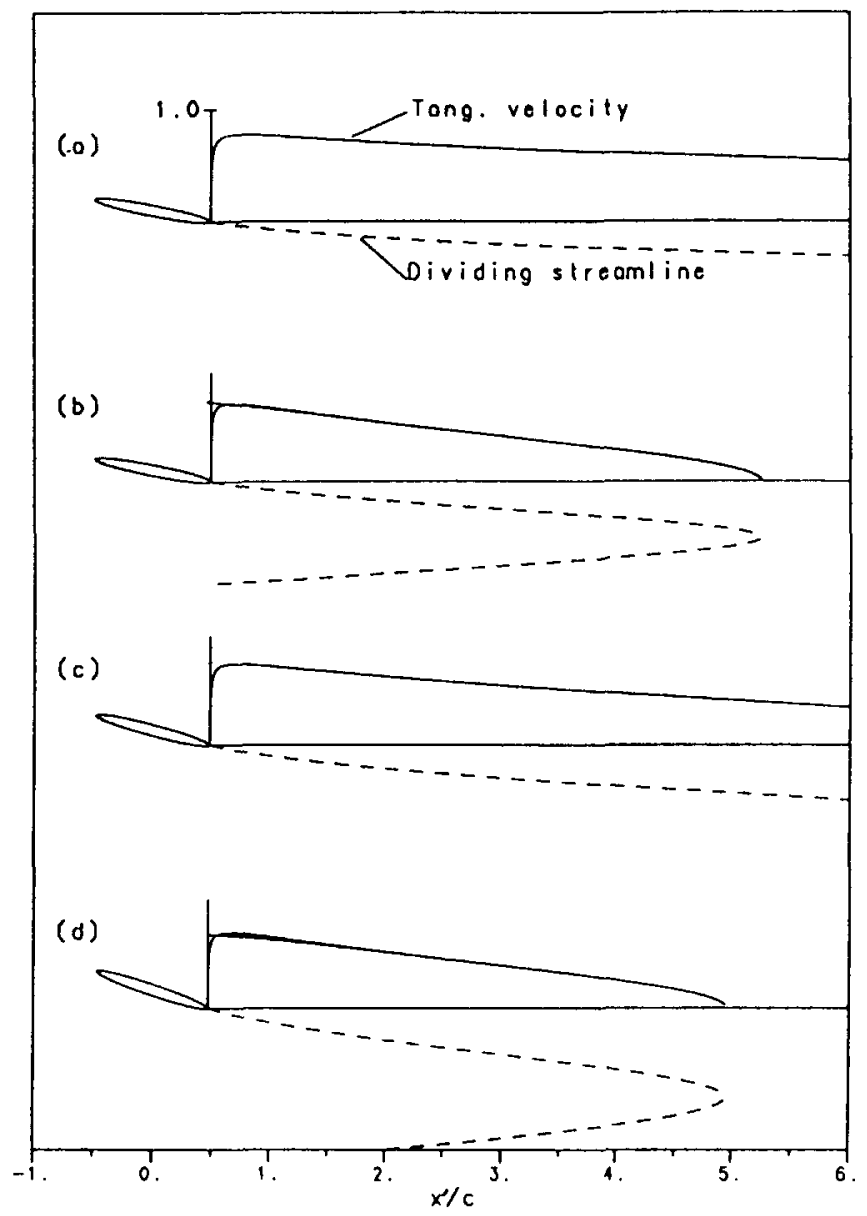

Fig. 5. Comparison of dividing trailing-edge streamlines and tangential velocity distributions along it for various flow conditions about a $10 \%$ ellipse. (a) $\alpha=12^{\circ}, K=1$; (b) $\alpha=12^{\circ}, K=1.6$; (c) $\alpha=16^{\circ}, K=1$; (d) $\alpha=20^{\circ}, K=1$.

likely to be little influenced by foil section shape (but are influenced by the parameters of the onset flow such as a velocity gradient and angle of attack as illustrated in fig. 5). The trailing-edge dividing streamlines in fig. 5 show that in some cases the "swirl" region of fig. 3 encompasses the foil within a closed streamline of finite extent.

In fig. 6 the disturbance potential on the surface of an ellipse of $10 \%$ thickness ratio at an angle of attack of $10^{\circ}$ is given for both uniform potential flow $(K=0)$ and uniformly sheared onset flow $(K=1)$. Differentiation of the potentials plus the addition of an appropriate component of the onset flow produces the surface speeds of figure 7 . These speed distributions are generally similar but the maximum surface speed is greater for sheared onset flow as is the lift coefficient (with nearly the same moment coefficient for the present case) as shown in fig. 8. These results are similar to those found by Tsien (1943) for the Joukowsky transformation but are more easily computed.

\section{Solution for non-uniform shear by field analysis}

For a non-uniform sheared onset flow, the condition that the disturbance be potential is invalid and eq. (1) governing the vorticity field must be explicitly solved. There are no elegant 


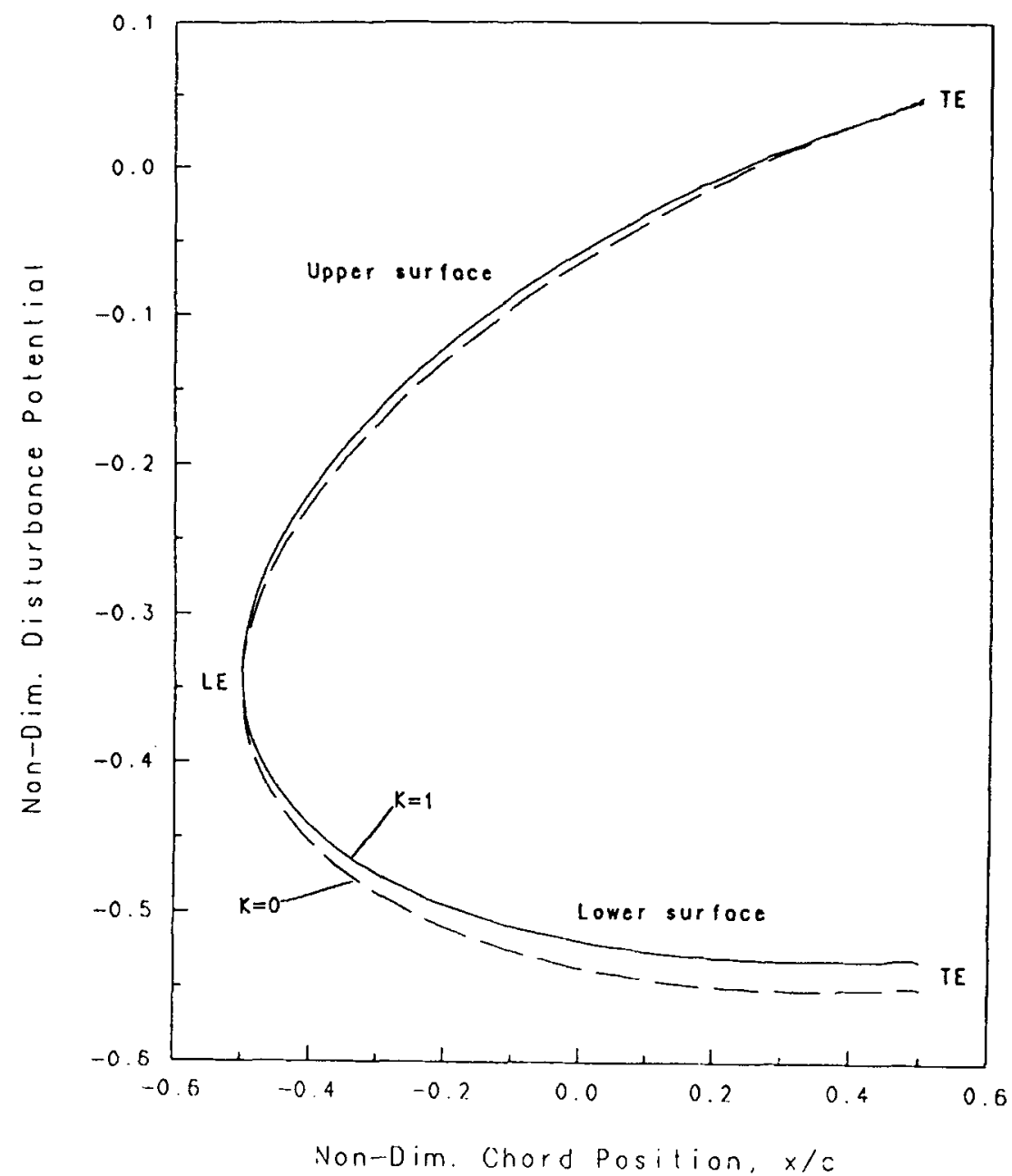

Fig. 6. Disturbance potential on an ellipse $(\tau=0.10)$ in uniform $(k=0$; broken line $)$ and shear $(K=1$; solid line $)$ flow at $\alpha=10^{\circ}$.

procedures available for defining solutions to this equation as there are in potential flow. A popular solution technique is a finite-difference or finite-element/finite-volume algorithm iterated over a field grid. There are two (nearly) independent aspects of this solution procedure: the specification of a grid and the solution of the field equations upon it. Herein we select a simple algebraic grid that is a mapping (using the Moriya transformation) of an easily defined grid in the circle plane (we will define this grid in detail in a following paragraph) and use a time-marching procedure to solve the field equations on this grid. The solution of eq. (1) and an accompanying determination of velocity components is called the vorticity-velocity approach. Alternatives are the velocity-pressure (or primitive variable) approach and the vorticity-streamfunction approach (see Chow et al., 1970, and Vooren, 1974, for similar problems with an alternative approach). Since we expect to eventually address the three-dimensional case, our interest is in only the first two approaches. We select the vorticity-velocity approach as the preferred procedure since it may be applied to non-inertial reference frames by simply adding terms to the boundary conditions for an algorithm that is proven for an inertial frame (Speziale, 1987; Suh, 1990). 


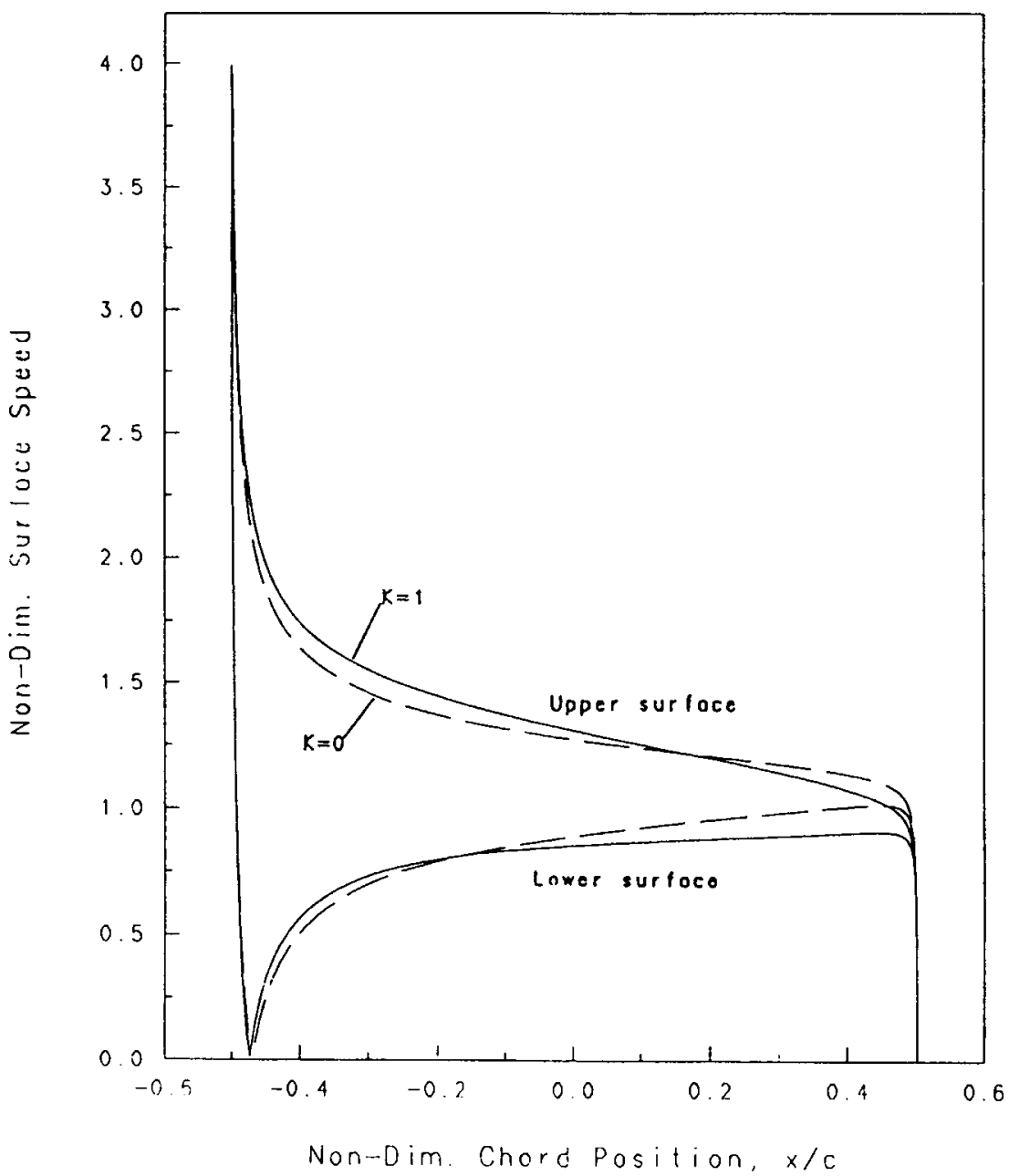

Fig. 7. Surface speed on ellipse of $10 \%$ thickness at $10^{\circ}$ angle of attack in potential and sheared onset flow.

The vorticity transport equation (1) is derived by taking the curl of both sides of the Euler equation governing the flow of an inviscid fluid. For two-dimensional flow the stretching term is eliminated and the simple-appearing expression results:

$$
\partial \omega / \partial t+\nabla \cdot(q \omega)=0
$$

The velocity field is determined by solving the kinematic relation $\omega \boldsymbol{k}=\boldsymbol{\nabla} \times \boldsymbol{q}$. This solution is indicated in eq. (11) and is a form of the Biot-Savart relation.

Since $u_{\mathrm{p}}$ and $u_{\mathrm{s}}$ are coupled in eq. (1) or (35), an iterative procedure results when each term is solved by separate numerical schemes, assuming that the other term is known or is already updated. The term $\boldsymbol{u}$ is determined uniquely by specified values of its normal component on the foil surface together with the tangential component of $\boldsymbol{u}_{\mathrm{s}}$ on the foil while the other boundary conditions are met by the assumed form of the solution in eq. (11). From the no-penetration condition the normal component of $\boldsymbol{u}$ must equal $-\boldsymbol{q}_{0} \cdot \boldsymbol{n}$ and we assume $\boldsymbol{u}_{\mathrm{s}}$ to be known or use its updated value during an iteration process. An integral equation similar to that of conventional boundary-element methods is found by taking the tangential 


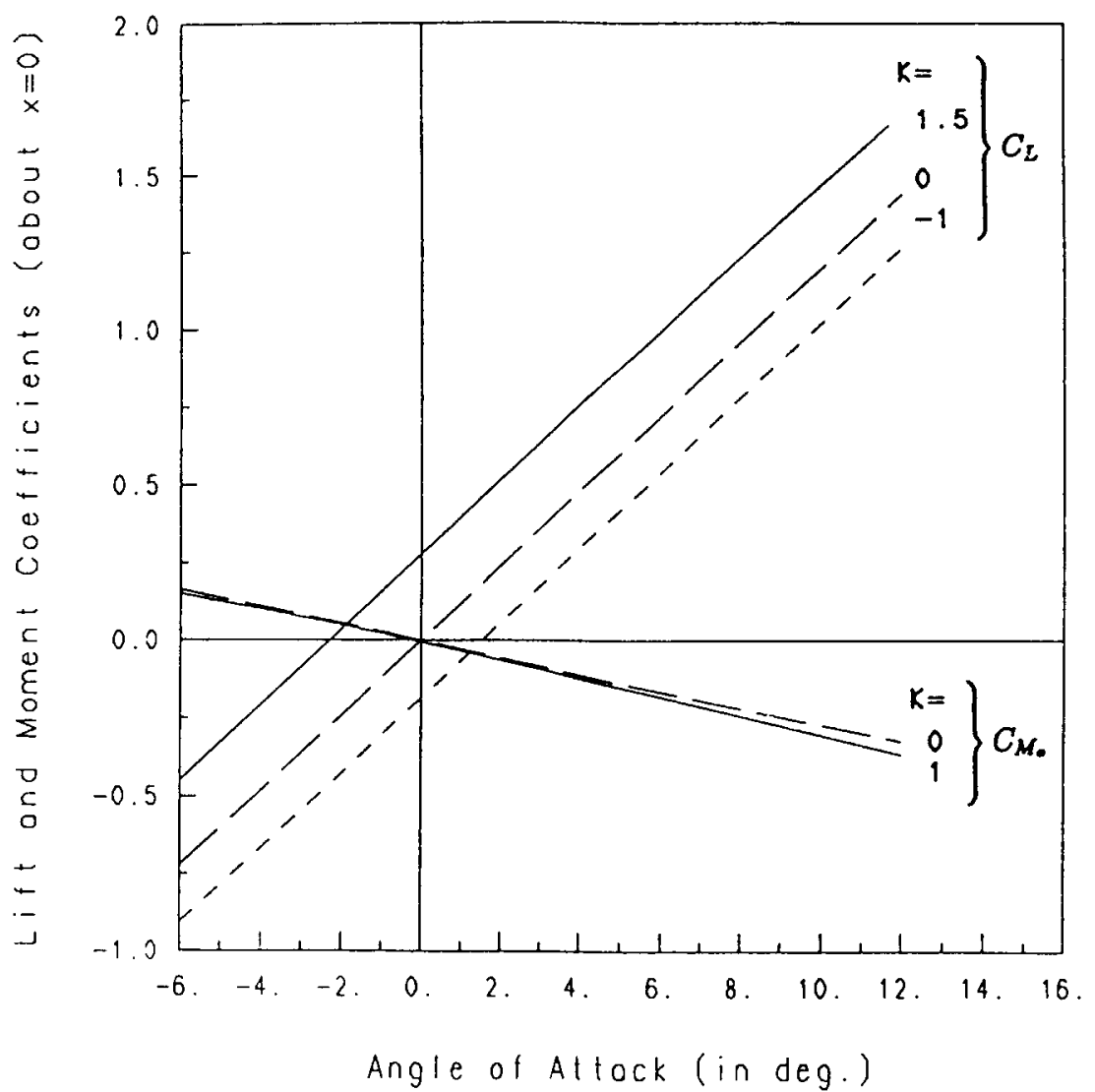

Fig. 8. Lift and moment coefficient (about midchord point) versus angle of attack for a Moriya foil $(\epsilon=0.05, \delta=0.1)$ in uniform flow.

component of the following equation

$$
\begin{aligned}
& u\left(x_{0}\right)-\frac{1}{\pi} f_{\mathrm{c}}[n(s) \times u(s)] \times \frac{x_{0}-s}{\left|x_{0}-s\right|^{2}} \mathrm{~d} l_{\mathrm{s}} \\
& \quad=\frac{1}{\pi} f_{\mathrm{c}} n(s) \cdot u(s) \frac{x_{0}-s}{\left|x_{0}-s\right|^{2}} \mathrm{~d} l_{\mathrm{s}}+\frac{1}{\pi} f f_{\mathrm{S}} \frac{\omega_{\mathrm{s}} \times\left(x_{0}-s\right)}{\left|x_{0}-s\right|^{2}} \mathrm{~d} S,
\end{aligned}
$$

where $\boldsymbol{x}_{0}$ is a point on the closed contour $\mathrm{c}$ and a doubling of the value on the surface results from the limiting process to define the Cauchy principal value (e.g., Brockett et al., 1989b). In the first iteration loop, we apply the no-penetration boundary condition expressed by the normal component of only the local undisturbed velocity and employ a potential-based boundary-element (panel) method approximation for $\boldsymbol{u}_{\mathrm{p}}$ (Suh, 1990).

The rotational component $\boldsymbol{u}_{\mathrm{s}}$ can be determined from the numerical algorithms for free vorticity governed by the vorticity transport equation (1) or (35). Once this distribution of disturbance vorticity $\omega_{\mathrm{s}}$ is determined, $\boldsymbol{u}_{\mathrm{s}}$ associated with $\omega_{\mathrm{s}}$ (i.e. $\omega_{\mathrm{s}} \boldsymbol{k}=\boldsymbol{\nabla} \times \boldsymbol{u}_{\mathrm{s}}$ ) is computed from the Biot-Savart integral:

$$
u_{\mathrm{s}}(x)=\frac{1}{2 \pi} \iint_{\mathrm{S}} \frac{\omega_{\mathrm{s}}(s) \times(x-s)}{|x-s|^{2}} \mathrm{~d} S,
$$

where the surface integral is taken over the region occupied by the fluid. 
In two-dimensional flow, the vorticity associated with a material element is constant. The vorticity change is due to only convection by the fluid. The vorticity transport equation given by (35) is a conservative form of a partial differential equation which makes it possible to use a finite-volume formulation. In the finite-volume formulation, the steady state is achieved when the net flux into a finite volume cell is equal to zero. After integrating the vorticity transport equation (35) over an arbitrary but stationary cell A with a cell boundary $\partial \mathrm{A}$ and then applying the divergence theorem one obtains the desired conservative form:

$$
\frac{\partial}{\partial t} \iint_{\mathrm{A}} \omega \mathrm{d} A+\int_{\partial \mathrm{A}} \boldsymbol{q} \cdot \boldsymbol{n} \omega \mathrm{d} l=0
$$

where $q \cdot n \boldsymbol{\omega}$ is the outward flux of $\boldsymbol{\omega}$ across the cell boundary. We approximate the vorticity transport equation as a discrete difference (actually volume) form for both time and space coordinates, by replacing the boundary integral by the sum over the four cell sides of a quadrilateral and using an explicit scheme for time marching:

$$
\omega_{i j}^{n+1}=\omega_{i j}^{n}-\frac{\Delta t_{i j}}{A_{i j}} \sum_{l=1}^{4} F_{l},
$$

where $\omega_{i j}^{n}$ is considered the average value of $\omega$ over cell $A_{i j}$ at the $n$th time step and $F_{l}$ represents the value of the flux outgoing throughout the $l$ th side of each cell, i.e. $F_{l}=$ $(\boldsymbol{q} \cdot \boldsymbol{n})_{l} \omega_{l} L_{l}$ where $n_{l}$ is the outward unit normal vector on the $l$ th side of the cell which has an arc length $L_{l}$. Choosing among a variety of expressions for the flux value, in the present work we take it to be the average along the side. The subscripts $i j$ refer to the ( $i$ th, $j$ th) cell and $\Delta t_{i j}$ is the local time increment for the cell $i j$. An O-type mesh is constructed with points in the physical plane corresponding to the intersection of constant angular lines and concentric circles in the mapping (circle) plane, by using the Moriya transformation as previously specified, similar to Shen (1977). A regular pattern of quadrilateral cells that divides the fluid region about the Moriya foil is illustrated in fig. 9. The grid in the circle plane is a series of radial lines and circles:

$$
\theta_{j}=\frac{2 \pi(i-1)}{N_{I}-1}, \quad r_{j}=1+\left(R_{\zeta}-1\right)\left[1-\cos \left(\frac{\pi(j-1)}{2\left(N_{J}-1\right)}\right)\right],
$$

where $N_{I}$ is the number of grid lines in the circumeferential direction, $N_{J}$ in the radial direction, and $R_{\zeta}$ is the radius in the mapping plane corresponding to the outer boundary of the computational domain (at nearly constant radius $R_{0}$ ). Since the first and last $i$-index coincide, a periodic boundary condition is applied along the interface corresponding to that index. Also on the foil surface $j=1$ (i.e. $r=1$ in the mapping plane), we set the vorticity convection, $(\boldsymbol{q} \cdot \boldsymbol{n})_{l} \boldsymbol{\omega}_{l}$, equal to zero from the no-penetration boundary condition. The far-field condition is applied simply by setting the vorticity on $R_{0}$ to that of the undisturbed flow.

The computation of the numerical fluxes $F_{l}$ in eq. (39) is expressed with an upwind first-order accurate scheme. The flux $F_{l}$ depends on the sign of the normal velocity conponent at the interface between two neighboring cells, designated by $I$ (inside) and $O$ (outside) respectively, as follows (Roache, 1972):

$$
\begin{aligned}
& F_{l}=(\boldsymbol{q} \cdot \boldsymbol{n})_{l} L_{l} \omega^{x}, \\
& \boldsymbol{\omega}^{x}=\omega_{\mathbf{I}} \quad \text { if }(\boldsymbol{q} \cdot \boldsymbol{n})_{l}>0, \quad \boldsymbol{\omega}^{x}=\omega_{\mathrm{O}} \quad \text { if }(\boldsymbol{q} \cdot \boldsymbol{n})_{l}<0 .
\end{aligned}
$$

The net mass flux $\left(\sum_{l=1}^{4}(\boldsymbol{q} \cdot \boldsymbol{n})_{l} L_{l}\right)$ at each cell should be zero to satisfy the continuity equation. In a discrete numerical version, the value of this divergence may not exactly zero at each cell, especially near the LE and the TE of the foil where this non-zero value (even if very 


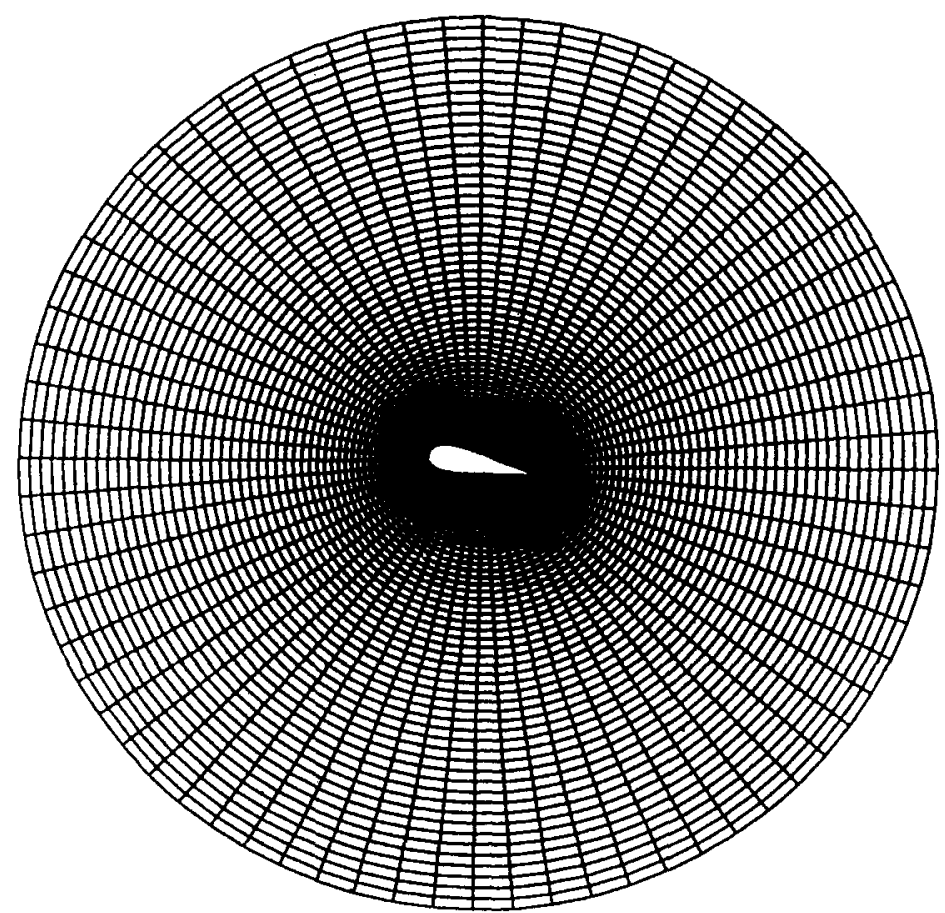

Fig. 9. An O-type grid generation about a Moriya foil $(\epsilon=0.1, \delta=0.4)$ at $10^{\circ}$ angle of attack with mesh $73 \times 61$; $R_{0}=4.0$.

small) may cause significant error in solving the vorticity transport equation for a large time step. To use the divergence-free condition to reduce this error, the mass fluxes through four sides of a cell are adjusted by adding (or subtracting) a part of the non-zero value to each side (with a weighting factor proportional to the magnitude of each flux). A practical local stability criterion (CFL condition) for the upwind scheme we use is deduced approximately from von Neumann stability analysis (Anderson et al., 1984):

$$
\left.\left(\Delta t_{i j} / d_{i j}\right) \sqrt{\left(q_{x}^{2}+q_{y}^{2}\right)}\right|_{i j} \leq 1,
$$

where $d_{i j}$ is the characteristic length equal to (the cell area)/(the longer diagonal of the cell). Near the stagnation points where the local time increment becomes exceptionally large, the above criterion is replaced by a cruder one (i.e. $\Delta t_{i j} / d_{i j} \leq 1$ ). The reason we use this local CFL condition is to reduce the computing time necessary to obtain a converged steady-state solution.

Next the velocity distribution is computed by evaluating the Biot-Savart integral (eq. 37) for the vorticity distribution obtained at the current iterative stage. The vorticity values given at the cell centers can be interpolated to those at the cell vertices (or extrapolated to boundaries so as to specify the values along the foil surface). Then the vorticity change $\omega_{\mathrm{s}} \boldsymbol{k}=\boldsymbol{\nabla} \times \boldsymbol{u}_{\mathrm{s}}$ due to the foil disturbance is obtained by subtracting the undisturbed component $\left(\omega_{0}\right)$ from the total vorticity magnitude $(\omega)$. If we approximate this disturbance vorticity distribution over a triangular element composed of three cell vertices (one of two triangular elements obtained by dividing each cell by its diagonal) as a bilinearly varying distribution, the Biot-Savart integral is evaluated by using an explicit closed-form equation similar to results presented by Webster (1975). The lengthy expressions employed are given by Suh (1990). 
To achieve fast convergence of the iteration, it is also necessary to employ a relaxation technique for the convection velocity $q$ according to:

$$
\boldsymbol{q}^{N}=\boldsymbol{q}^{0}+r_{q}\left(\boldsymbol{q}^{U}-\boldsymbol{q}^{0}\right)
$$

Here, $\boldsymbol{q}^{N}$ is the value calculated from the numerical procedure at the present iteration, $\boldsymbol{q}^{0}$ is the value obtained by application of this formula at the previous iteration, $q^{U}$ is the formally predicted value of the convection velocity at the present iteration loop, and $r_{q}$ is the relaxation parameter. The convergence of the iteration process of the velocity and vorticity calculation is measured with the difference of surface speed between two successive iterations. The tolerance of the local maximum difference (i.e. the infinite norm) for the iteration was typically set at 0.01 . The first iteration results correspond to those of conventional potential-flow calculation for the disturbance velocity, namely neglecting, $\boldsymbol{u}_{\mathrm{s}}$, the rotational term of the disturbance velocity. We find a significant change in the velocity distribution when including the rotational term. Figure 10 presents the numerical results obtained by applying the present method to a Moriya foil $(\epsilon=0.1$ and $\delta=0.4)$ in what we call a more realistic flow condition. The undisturbed velocity is specified as $\boldsymbol{q}_{0}=\left(1+\tanh y^{\prime}\right) \boldsymbol{i}^{\prime}$ with an angle of attack $\alpha=10^{\circ}$. Its vorticity magnitude at the midchord is 1 , similar to the uniformly sheared case with $K=1$. The Kutta condition is applied for this calculation, including the rotational disturbance component when the panel-method approximation is employed. The desired tolerance is obtained after 8 iterations with a mesh $73 \times 61$ ( 72 by 60 cells, as shown in fig. 9) and the relaxation parameter $r_{q}=0.25$. A field with $R_{0}$ equal to about half that of fig. 9 and with about half the contours encircling the foil produced nearly identical results. The difference between the uniform-shear calculation (denoted by a solid line) and the numerical shear-flow field calculation (denoted by the small circles) is the influence of the variable vorticity. The non-uniform shear-flow solution indicates surface speeds generally similar in shape to the potential-flow case, as well as generally similar to the uniformly sheared onset flow case, which is also shown in fig. 10. The non-linear (more realistic) sheared onset flow produces a non-dimensional surface speed that has a peak value (reducing by 0.04 relative to the uniform-shear case) about midway between the uniform-onset and uniform-shear case. On the pressure or lower side of the foil, the numerical solution is nearly identical with the uniform-shear case. The lift is also about midway between the two analytic cases. Greater details of this solution as well as other examples are available in Suh (1990). These numerical results tend to support the experimental data of Payne and Nelson (1985) for a foil experiment in a tunnel for which the shear flow influences the pressure but with a reduced effect on the loads. In particular, the results in fig. 10 show a reduced lift coefficient with a locally sheared onset field.

\section{Summary}

For uniform shear, the disturbance to the onset flow is potential and an exact solution can be defined by a conformal mapping of the flow about a lifting circular cylinder. The Moriya transformation with terms to order $1 / z^{2}$ is chosen as it has a parameter in the transformation that can be used to set a fixed chordlength and produce equations for the flow speed on the profile that can be readily computed at specified points. The trends for lift and surface speed are similar to results found previously by others: generally a shift in angle of zero lift and an increase in surface speed on the side of the profile with increased local onset speed. The body streamline may be a closed contour within the field. The results obtained reveal general trends and also provide easily accessible quantitative values of surface speed and loads useful in validating numerical solutions. 


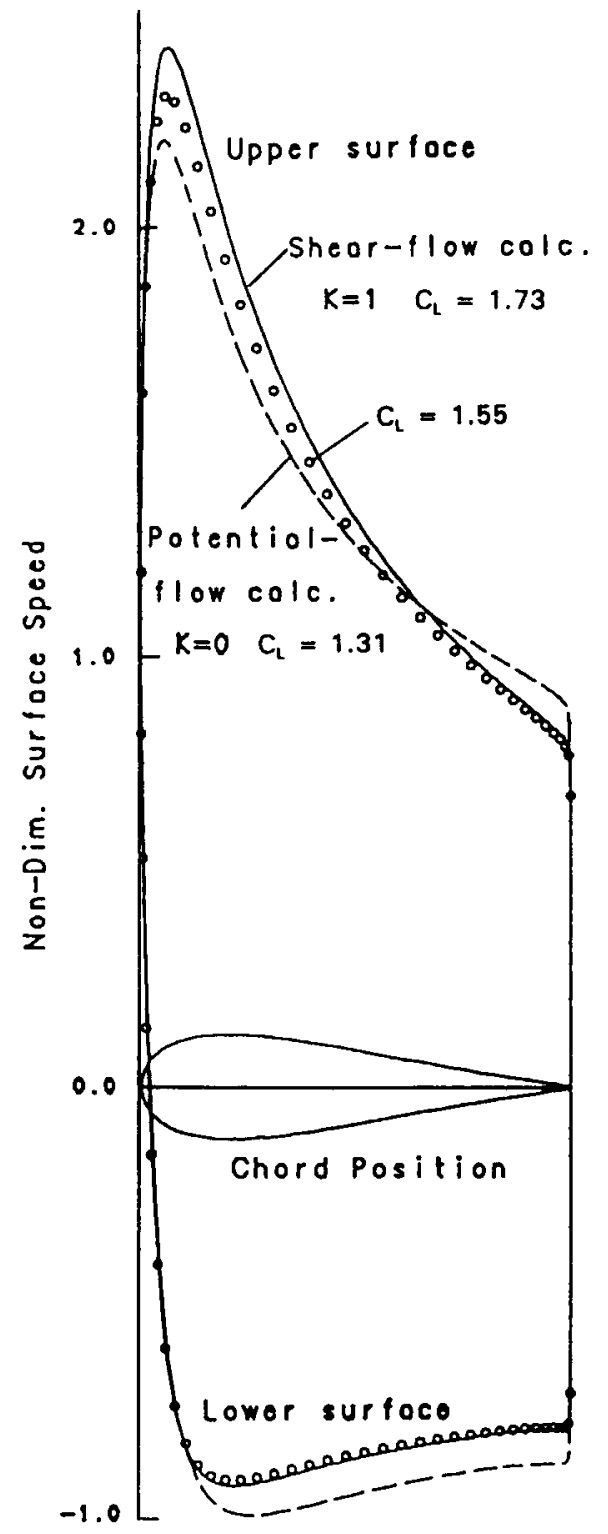

Fig. 10. Surface speed on a Moriya profile $(\epsilon=0.1, \delta=0.4)$ for three onset velocity profiles at $10^{\circ}$ angle of attack.

For non-linear shear, a numerical solution of the field equations for the vorticity-velocity formulation has been undertaken using a field grid derived with the conformal transformation of concentric circles and rays in the circle plane. An iterative time-marching approach has been selected and a solution is marched from an initial potential representation of the disturbance velocity (i.e. $\boldsymbol{u}_{\mathrm{p}}(\boldsymbol{x}, t)$ and $\left.\omega_{\mathrm{s}}(\boldsymbol{x}, t)\right)$ to a time-invariant solution for the disturbance (i.e. $\left.\boldsymbol{u}_{\mathrm{p}}(\boldsymbol{x}, \infty)+\boldsymbol{u}_{\mathrm{s}}(\boldsymbol{x}, \infty), \boldsymbol{\omega}_{\mathrm{s}}(\boldsymbol{x}, \infty)\right)$ for which the velocity field consists of appropriate potential and rotational components. A numerical example is presented that is locally similar to one of the solutions from the uniform shear case: the profile shape is identical and the onset flow gradient is identical along the onset flow line that passes through the midchord point but the onset shear flow tends to constant values away from the profile. The lift increment due to 
shear is reduced to about half that for the uniform-shear case and the maximum surface-speed shear increment is also reduced to about half the increment for the uniform-shear case.

These results suggest that the uniform-shear solution will overestimate the influence of shear in practical cases for which only a limited region of the onset flow field is sheared.

\section{References}

Anderson, D.A. et al. (1984) Computational Fluid Mechanics and Heat Transfer (Hemisphere, Washington).

Ashley, H. and M. Landahl (1965) Aerodynamics of Wings and Bodies (Addison-Wesley, Reading).

Atassi, H.M. (1984) The Sears problem for a lifting airfoil revisited - new results, J. Fluid Mech. 141, $109-122$.

Brockett, T. (1986) Propeller interaction with an axisymmetric sheared onset flow, Proc. 16th Symp. on Nalal Hydrodynamics (National Academy Press) pp. 268-288.

Brockett, T. (1989a) Some exact results for plane incompressible flow, Fluid Dyn. Res. 5, 111-116.

Brockett, T.E. et al. (1989b) Limiting forms for surface singularity distributions when the field point is on the surface, J. Eng. Math. 23, 53-79.

Chow, F. et al. (1970) Numerical investigation of an airfoil in a non-uniform stream, J. Aircraft 7 (6), 531-537.

Joukowsky, N.E. (1910) Über die Konturen der Tragflächen der Drachenflieger, Z. Flugtech. Motorluftsch. 1, 281-284.

Moriya, T. (1938) A method of calculation aerodynamic characteristics of an arbitrary wing section, J. Soc. Aeron. Sci. Japan 5 (33), 7-17 (in Japanese).

Moriya, T. (1941) On the aerodynamic characteristic of an arbitrary wing section, J. Soc. Aeron. Sci. Japan 8, (78), 1054-1060 (in Japanese, English translation in: Selected Scientific and Technical Papers, University of Tokyo, 1959).

Murray, J.D. and A.R. Mitchell (1957) Flow with variable shear past circular cylinders, Q. J. Mech. Appl. Math. 10, $13-23$.

Payne, F.M. and R.C. Nelson (1985) Aerodynamic characteristics of an airfoil in a nonuniform wind profile, $J$. Aircraft 22 (1) , 5-10.

Roache, P.J. (1972) Computational Fluid Dynamics (Hermosa Pub., Albuquerque).

Shen, S.F. (1977) An aerodynamicist looks at the finite element method, Finite Elements Fluids 2, $179-204$.

Speziale, C.G. (1987), On the advantage of the vorticity-velocity formulation of the equations of fluid dynamics, $J$. Comp. Phys. 73, 476-480.

Suh, J.-C. (1990) Unsteady analysis for a two-dimensional foil in uniformly sheared onset flow, PhD Dissertation, University of Michigan.

Tsien, H.-S. (1943) Symmetrical Joukowsky airfoils in shear flow, Q. Appl. Math. 1, 130-148.

Vooren, H. and Th.E. Labrujere (1974) Finite element solution of the incompressible flow over an airfoil in a non-uniform stream, in: Numerical Methods in Fluid Dynamics, eds. C.A. Brebbia and J.J. Conner (Pentech Press) pp. 23-41.

Webster, W.C. (1975), The flow about arbitrary, three-dimensional bodies, J. Ship. Res. 19 (4), $206-218$.

Yih, C.-S. (1969) Fluid Mechanics (McGraw-Hill, New York). 